\title{
A cache-aware performance prediction framework for GPGPU computations
}

The 8th Workshop on UnConventional High Performance Computing 2015

Alexander Pöppl, Alexander Herz

August 24th, 2015 


\section{Agenda}

\section{Introduction}

Motivation

Contributions

Example

Model

Execution Time Computation

Memory Transfer

Empty Kernels

Workgroup Size

Basic Operations

Memory accesses

\section{Evaluation}

Qualitative Evaluation

Quantitative Evaluation

\section{Further Work}




\section{Introduction}

\section{Motivation}

- OpenCL is used for running heterogeneous HPC applications

- It is low level, fairly explicit, and has manual task management

\footnotetext{
${ }^{1}$ Cédric Augonnet et al. "StarPU: A Unified Platform for Task Scheduling on Heterogeneous Multicore Architectures". English. In: Euro-Par 2009 Parallel Processing. Ed. by Henk Sips, Dick Epema, and Hai-Xiang Lin. Vol. 5704. Lecture Notes in Computer Science. Springer Berlin Heidelberg, 2009, pp. 863-874. ISBN: 978-3-642-03868-6. DOI: 10.1007/978-3-642-03869-3_80. URL: http://dx.doi.org/10.1007/978-3-642-03869-3_80.

2 Gregory F. Diamos and Sudhakar Yalamanchili. "Harmony: An Execution Model and Runtime for Heterogeneous Many Core Systems". In: Proceedings of the 17th International Symposium on High Performance Distributed Computing. HPDC '08. Boston, MA, USA: ACM, 2008, pp. 197-200. ISBN: 978-1-59593-997-5. DOI: 10.1145/1383422.1383447. URL: http://doi.acm.org/10.1145/1383422.1383447.
} 


\section{Introduction}

\section{Motivation}

- OpenCL is used for running heterogeneous HPC applications

- It is low level, fairly explicit, and has manual task management

- Hence runtime systems with schedulers, such as StarPU ${ }^{1}$ or Harmony ${ }^{2}$ have been developed

- These schedule tasks onto heterogeneous hardware based on expected runtime.

- High-quality estimations crucial for efficient schedules.

\footnotetext{
${ }^{1}$ Cédric Augonnet et al. "StarPU: A Unified Platform for Task Scheduling on Heterogeneous Multicore Architectures". English. In: Euro-Par 2009 Parallel Processing. Ed. by Henk Sips, Dick Epema, and Hai-Xiang Lin. Vol. 5704. Lecture Notes in Computer Science. Springer Berlin Heidelberg, 2009, pp. 863-874. ISBN: 978-3-642-03868-6. DOI: 10.1007/978-3-642-03869-3_80. URL: http://dx.doi.org/10.1007/978-3-642-03869-3_80.

2 Gregory F. Diamos and Sudhakar Yalamanchili. "Harmony: An Execution Model and Runtime for Heterogeneous Many Core Systems". In: Proceedings of the 17th International Symposium on High Performance Distributed Computing. HPDC '08. Boston, MA, USA: ACM, 2008, pp. 197-200. ISBN: 978-1-59593-997-5. DOI: 10.1145/1383422.1383447. URL: http://doi.acm.org/10.1145/1383422.1383447.
} 


\section{Introduction}

\section{Motivation}

- Performance Prediction models already exist, and work well with earlier GPU architectures.

- Introduction of Caches complicate predictions.

- GPU memory Hierarchy needs to be considered. 


\section{Introduction}

\section{Contributions}

- Categorization of memory accesses into classes with distinct performance characteristics.

- Fully static OpenCL computation prediction model.

- Evaluation using randomly generated OpenCL kernels shows that a cache-aware model improves predictions. 


\section{Introduction}

\section{Example}

- Popular operation: Stencil operations

- Array of size: $n * m$

$$
b(i, j)=a(i, j)^{2}-a(1, j)
$$




\section{Introduction}

\section{Example}

1: $n_{\mathrm{Wl}}=m * n$

2: mem $_{\mathrm{GPU}}^{\text {input }} \leftarrow$ device.alloc $\left(n_{\mathrm{WI}} * s_{\mathrm{WI}}\right)$

3: mem $_{G P U}^{\text {output }} \leftarrow$ device.alloc $\left(n_{\mathrm{WI}} * s_{\mathrm{WI}}\right)$

4: copyDataToGPU $\left(\rightarrow\right.$ mem $\left._{G P U}^{\text {input }}\right)$

5: device.kernel $\left(n_{\mathrm{WI}}, n_{\mathrm{WG}}, m, n\right)$ $\Rightarrow \forall i d \in\left\{0, . ., n_{\mathrm{WI}}\right\} . s q \_\bmod \left(\operatorname{mem}_{\mathrm{GPU}}^{\text {input }}\right.$, mem $\left._{\mathrm{GPU}}^{\text {output }}, m, n\right)$

6: copyDataFromGPU $\left(\rightarrow\right.$ mem $\left._{G P U}^{\text {output }}\right)$ 


\section{Introduction}

\section{Example}

kernel void sq_mod(global float * matrix, global float $*$ res,

unsigned int $\mathrm{m}$, unsigned int $\mathrm{n})\{$

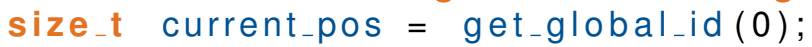
unsigned int current_row $=$ current_pos $/ n$; unsigned int current_col = current_pos $\% n$; res $[$ current_pos $]=$ matrix [ current_row $* n$ + current_col]

* matrix [ current_row $* \mathrm{n}+$ current_col]

- matrix[ current_col]; 


\section{Model}

\section{Execution Time Computation - Computation of the Runtime}

$$
\begin{aligned}
& t\left(n_{\mathrm{WI}}, s_{\mathrm{WI}}, n_{\mathrm{WG}}\right)=t_{\text {Transfer }}\left(n_{\mathrm{WI}}, s_{\mathrm{WI}}\right)+t_{\text {Kernel }}\left(n_{\mathrm{WI}}, n_{\mathrm{WG}}\right) \\
& t_{\text {Kernel }}\left(n_{\mathrm{WI}}, n_{\mathrm{WG}}\right)=\frac{t_{\text {Base }}\left(n_{\mathrm{WI}}\right)+\sum_{\mathrm{Op} \in \text { Expr.-Types }} W_{\mathrm{Op}}\left(n_{\mathrm{Op}}\right) t_{\mathrm{Op}}\left(n_{\mathrm{WI}}\right)}{U\left(n_{\mathrm{WG}}, n_{\mathrm{XU}}\right)}
\end{aligned}
$$

$n_{\mathrm{WI}} \quad$ Number of work-items

$n_{\text {WG }} \quad$ Number of work-items per work-group

$S_{W I} \quad$ Size of a work-item in bytes

$n_{\mathrm{XU}} \quad$ Number of execution units on the GPU 


\section{Model}

Memory Transfer

- GPUs have a dedicated portion of memory for their computations

- Time for memory transfer governed by two variables

- bw Bandwidth

- Iprop Propagation latency 


\section{Model}

\section{Memory Transfer}

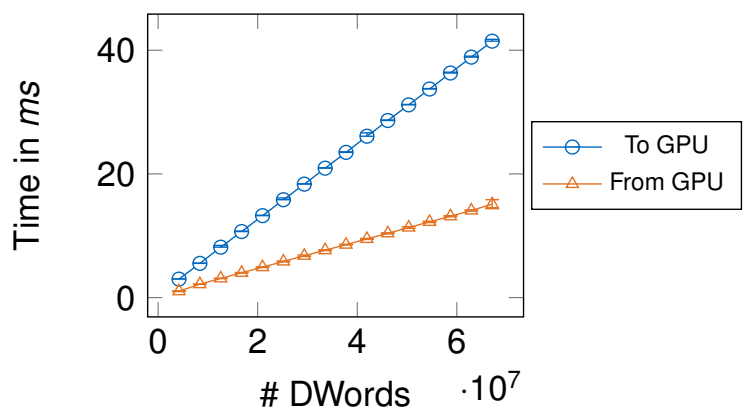

Figure: Memory Transfer times

- $t_{\text {trans }}^{\text {to }}\left(n_{\mathrm{WI}}\right)=b w_{\text {to }}^{-1} n_{\mathrm{WI}}+l_{\text {to }}$

- $t_{\text {trans }}^{\text {from }}\left(n_{\mathrm{WI}}\right)=b w_{\text {from }}^{-1} n_{\mathrm{WI}}+l_{\text {from }}$ 


\section{Model}

\section{Empty Kernels}

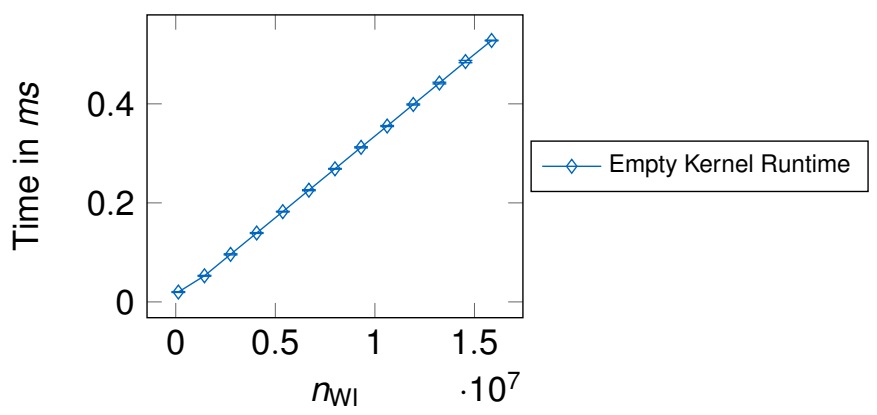

Figure: Execution times for empty kernels.

- $t_{\text {Base }}\left(n_{\mathrm{WI}}\right)=c_{\text {Base }} n_{\mathrm{WI}}+c_{\text {Base }}^{\text {fixed }}$ 


\section{Model}

Workgroup Size

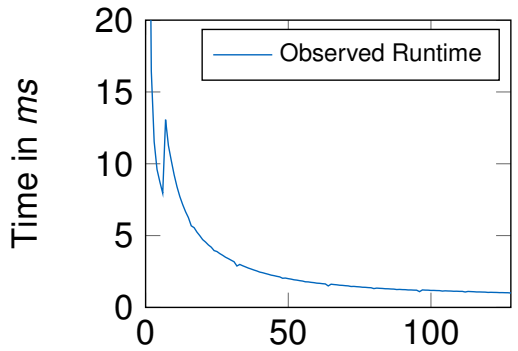

Work-items per work-group

(a) NVidia GT-650M

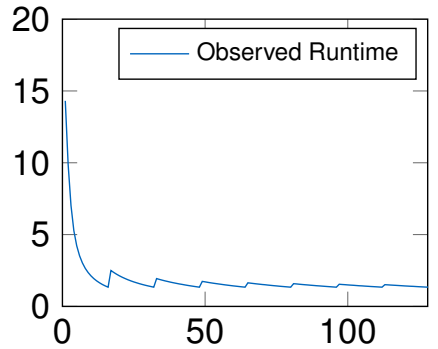

Work-items per work-group

(b) Intel HD Graphics 4000

Figure: Execution time for different work-group sizes. The kernel we used to evaluate this behavior performs one read from and write to the global memory, and one floating point division. 


\section{Model}

Workgroup Size - Modelling the behavior

- Periodic spikes in execution time.

- Especially visible on the HD 4000.

\section{Influence of Work-Group size}

$$
U\left(n_{\mathrm{WG}}, n_{\mathrm{XU}}\right)=\underbrace{\frac{\left\lfloor\frac{n_{\mathrm{WG}}}{n_{\mathrm{XU}}}\right\rfloor}{\left\lceil\frac{n_{\mathrm{WG}}}{n_{\mathrm{XU}}}\right\rceil}}_{A}+\underbrace{\frac{n_{\mathrm{WG}} \bmod n_{\mathrm{XU}}}{n_{\mathrm{XU}}} \frac{\left\lceil\frac { n _ { \mathrm { WG } } } { n _ { \mathrm { XU } } \rceil } \left\lfloor\left\lfloor\frac{n_{\mathrm{WG}}}{n_{\mathrm{XU}}}\right\rfloor\right.\right.}{\left\lceil\frac{n_{\mathrm{WG}}}{n_{\mathrm{XU}}}\right\rceil}}_{B}
$$




\section{Model}

\section{Basic Operations}
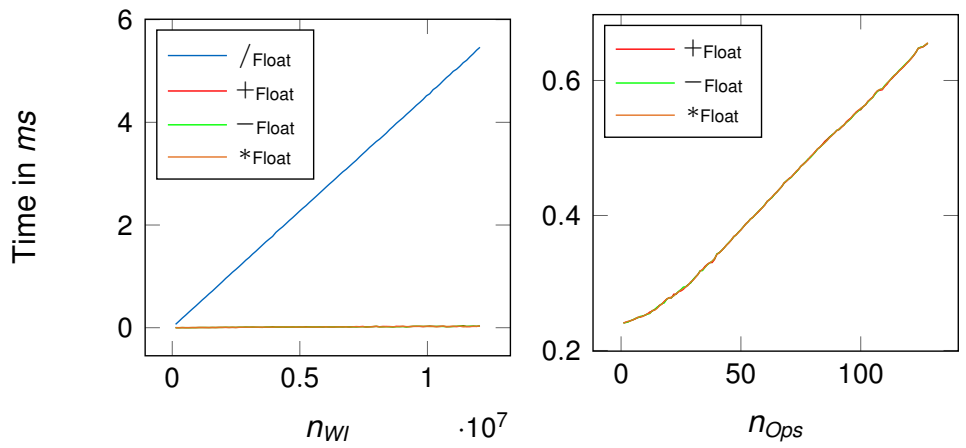

(a) One operation per work-item

(b) Multiple Operations per work-item

Figure: Progression of the execution time for basic operations. 


\section{Model}

\section{Basic Operations}

$$
\begin{gathered}
W_{\mathrm{op}}^{\mathrm{type}}\left(n_{\mathrm{Ops}}\right)= \begin{cases}a n_{\mathrm{Ops}}^{b}+c & : n_{\mathrm{Ops}} \leq n_{\mathrm{Ops}}^{\mathrm{sat}} \\
a^{\prime} n_{\mathrm{Ops}}+c^{\prime} & : n_{\mathrm{Ops}}>n_{\mathrm{Ops}}^{\mathrm{sat}}\end{cases} \\
t_{\mathrm{Op}}^{\mathrm{type}}\left(n_{\mathrm{WI}}\right)=c_{\mathrm{Op}}^{\mathrm{type}} n_{\mathrm{WI}}
\end{gathered}
$$

- $a, a^{\prime}, b, c, c^{\prime}$ are obtained by fitting $W_{\mathrm{op}}^{\text {type }}\left(n_{\mathrm{Ops}}\right)$ to $4 \mathrm{~b}$ - $c_{\mathrm{op}}^{\text {type }}$ is obtained by fitting $t_{\mathrm{op}}^{\text {type }}\left(n_{\mathrm{WI}}\right)$ to $4 \mathrm{a}$. 


\section{Model}

Memory accesses

- In OpenCL, 3 different kinds of memory accesses are available

- private: Used for local variables, parameters.

- local: Shared between work-items within a work-group

- global: Shared amongst all work-items

- Usually implemented using different kinds of memory. 


\section{Model}

\section{Memory accesses}

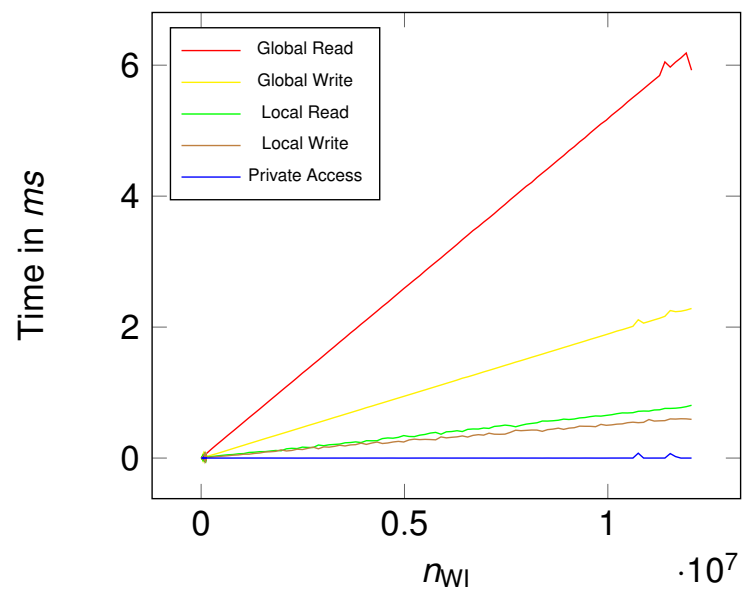

A. Pöppl, A. Herz: A cache-aware performance prediction framework for GPGPU computations UCHPC 2015, August 24th, 2015 


\section{Model}

\section{Memory accesses - Coalesced Accesses}

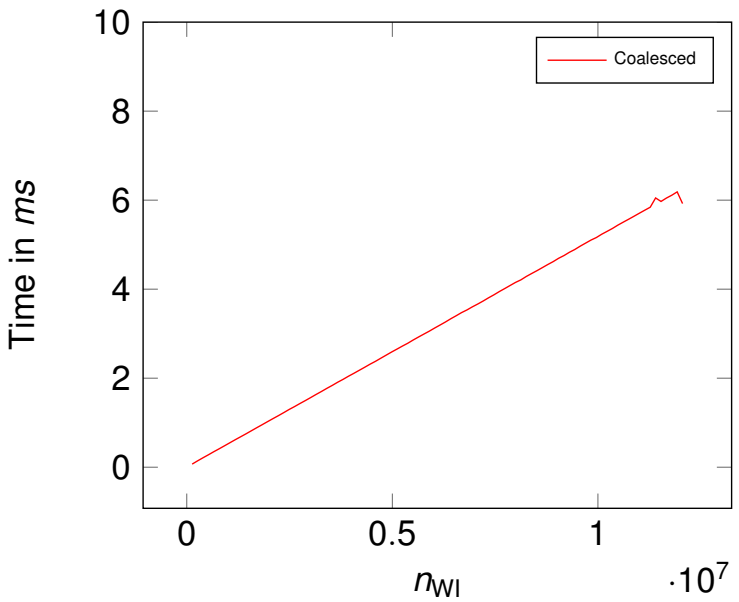

A. Pöppl, A. Herz: A cache-aware performance prediction framework for GPGPU computations UCHPC 2015, August 24th, 2015 


\section{Model}

\section{Memory accesses - Constant Accesses}

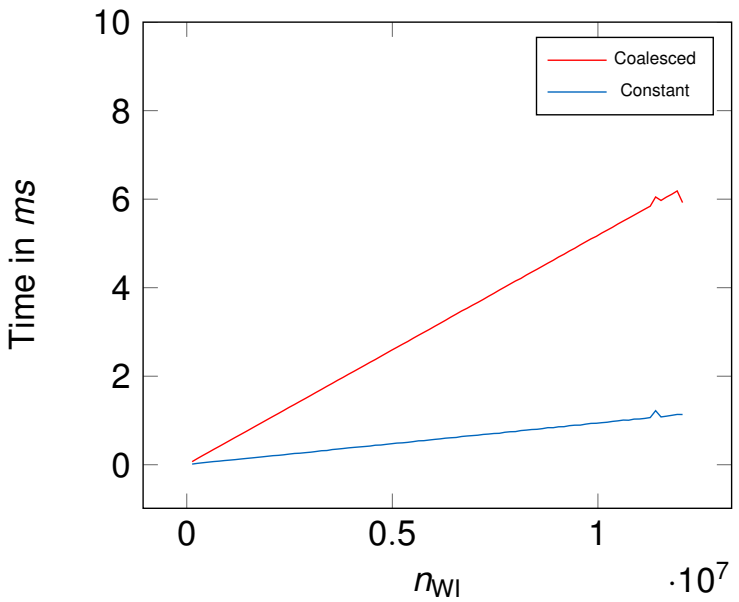

A. Pöppl, A. Herz: A cache-aware performance prediction framework for GPGPU computations UCHPC 2015, August 24th, 2015 


\section{Model}

Memory accesses - Interval Accesses

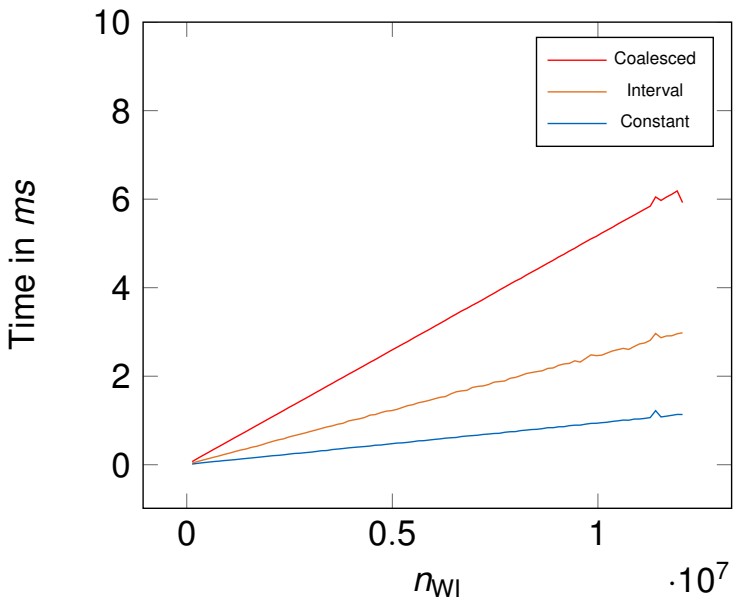

A. Pöppl, A. Herz: A cache-aware performance prediction framework for GPGPU computations UCHPC 2015, August 24th, 2015 


\section{Model}

\section{Memory accesses - Two Identical Accesses}

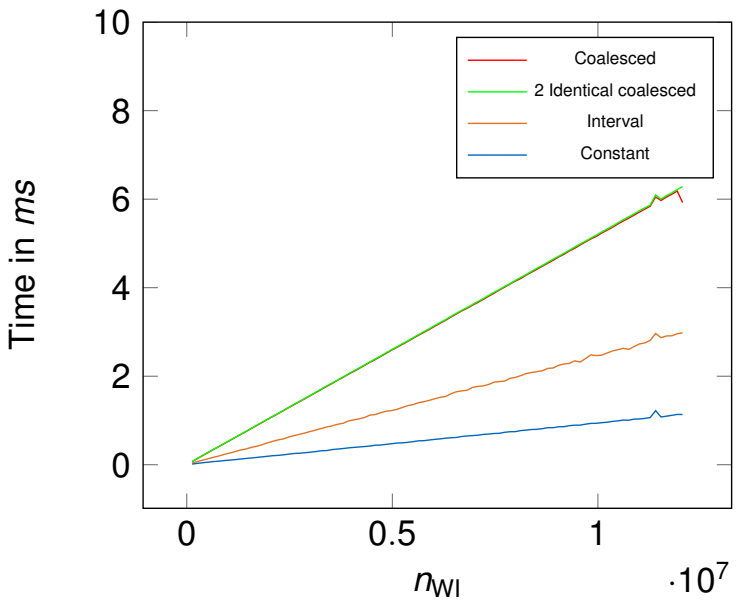

A. Pöppl, A. Herz: A cache-aware performance prediction framework for GPGPU computations UCHPC 2015, August 24th, 2015 


\section{Model}

\section{Memory accesses - Complex Accesses}

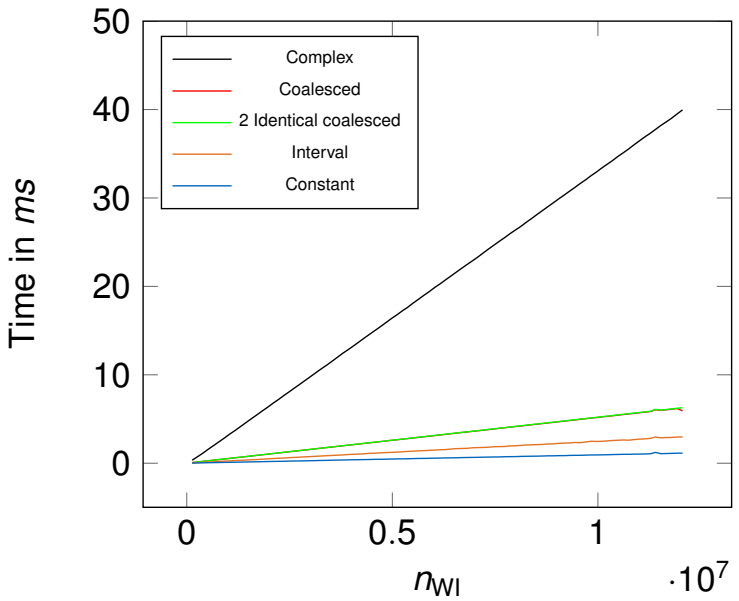




\section{Evaluation}

\section{Qualitative Evaluation}

- Static prediction of the execution time given the following data:

- Kernel Source Code

- Data about GPU characteristics

- Number of work-items $n_{\mathrm{WI}}$ 


\section{Evaluation}

\section{Qualitative Evaluation}

- Static prediction of the execution time given the following data:

- Kernel Source Code

- Data about GPU characteristics

- Number of work-items $n_{\mathrm{WI}}$

\begin{tabular}{rcl} 
Cost Type & \# in Kernel & Time in $\mu s$ \\
\hline$-_{\text {float }}$ & 1 & 74.16 \\
$*_{\text {float }}$ & 1 & 74.54 \\
+int $_{\text {int }}$ & 1 & 55.13 \\
$*_{\text {int }}$ & 7 & 81.04 \\
$/$ int & 4 & 1506 \\
private access & 1 & 0.0 \\
interval global read access & 1 & 770.9 \\
continuous global read access & 1 & 2335 \\
base cost & 1 & 3191 \\
\hline \hline work-group size & 1024 & - \\
\hline final prediction & & $\mathbf{8 0 8 9}$
\end{tabular}




\section{Evaluation}

\section{Qualitative Evaluation}

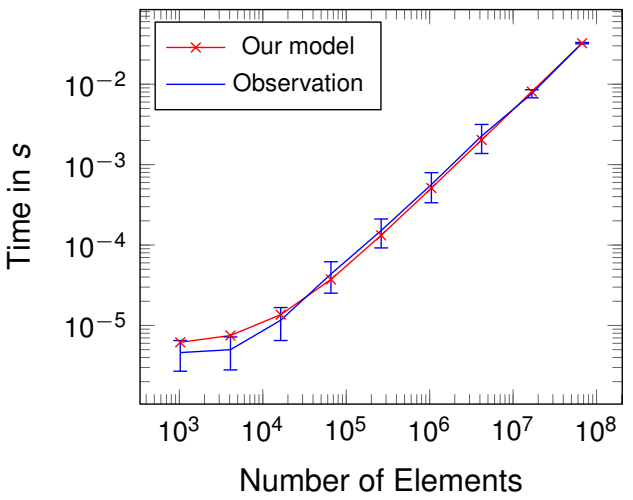

A. Pöppl, A. Herz: A cache-aware performance prediction framework for GPGPU computations UCHPC 2015, August 24th, 2015 


\section{Evaluation}

\section{Quantitative Evaluation}

- Quantitative evaluation through generated OpenCL Kernels

- 2 Sets of kernels, Unrestricted and "Realistic"

- Unrestricted Set

- Little restrictions on complexity

- Complex memory access patterns possible

- $((\operatorname{xxx}[((\mathrm{y}+\mathrm{x})+454) \& 0 \mathrm{x} 7 \mathrm{~F}] /(\operatorname{matrix}[\mathrm{x}][\mathrm{y}] * \mathrm{x}))-$ (matrix $[\mathrm{x}][\mathrm{y}]+(\operatorname{matrix}[\mathrm{x}][\mathrm{y}]+((\operatorname{matrix}[(44190 *(20+\mathrm{x})) \%$ HEIGHT] [1094 \% WIDTH] - $\mathrm{xxx}[71632 \&$ 0x7F] $)-((162.82883 f *(\mathrm{x}$ $-\mathrm{y}))+(785.19073 \mathrm{f} /(((((\operatorname{matrix}[\mathrm{x}][\mathrm{y}]-\operatorname{matrix}[\mathrm{x}][\mathrm{y}])-$ $\operatorname{xxx}[(\mathrm{y} * \mathrm{x}) \& 0 \mathrm{x} 7 \mathrm{~F}])+77.578835 f)+\operatorname{matrix}[\mathrm{x}][\mathrm{y}])+$ $550.7608 f())))))+\operatorname{xxx}[x \& 0 x 7 F]$

- Realistic Set

- Complexity restricted, limited number of nodes in syntax tree

- No overly complex memory access patterns

- ( $(\mathrm{x} /$ (xxx [x \& $0 \mathrm{x} 7 \mathrm{~F}]$ / (matrix [1 \% HEIGHT] [361 \% WIDTH] * $\operatorname{matrix}[\mathrm{x}][\mathrm{y}]))) * \operatorname{xxx}[\mathrm{y} \&$ Ox7F]) $+747.18744 \mathrm{f}$ 


\section{Evaluation}

\section{Quantitative Evaluation - GT-650M}

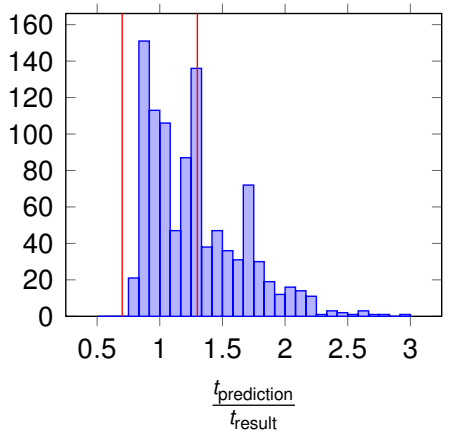

(a) Realistic Set

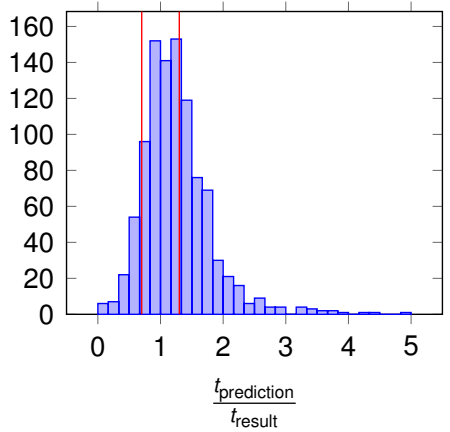

(b) Unrestricted Set

- $0.7<\frac{t_{\text {prediction }}}{t_{\text {result }}}<1.3$ for $63 \%$ of all samples for the restricted set. - $0.7<\frac{t_{\text {prediction }}}{t_{\text {result }}}<1.3$ for $50 \%$ of all samples for the unrestricted set. 


\section{Evaluation}

\section{Quantitative Evaluation - Quadro K4000}

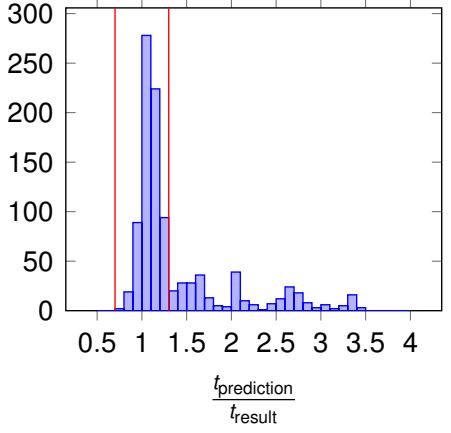

(c) Realistic Set

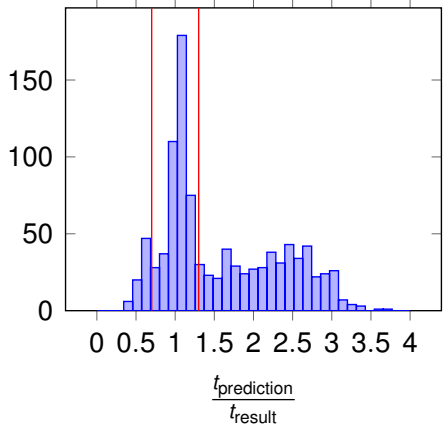

(d) Unrestricted Set

- $0.7<\frac{t_{\text {prediction }}}{t_{\text {result }}}<1.3$ for $71 \%$ of all samples for the restricted set.

- $0.7<\frac{t_{\text {prediction }}}{t_{\text {result }}}<1.3$ for $43 \%$ of all samples for the unrestricted set. 


\section{Evaluation}

\section{Quantitative Evaluation - Comparison}

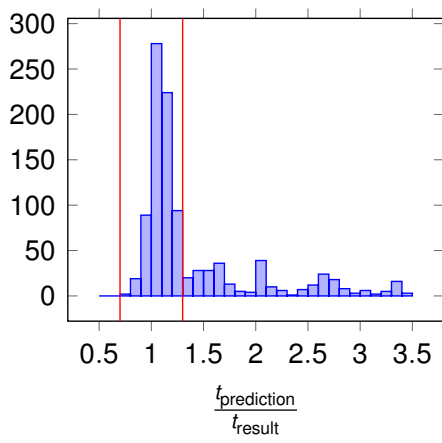

(e) Cache-Aware Model

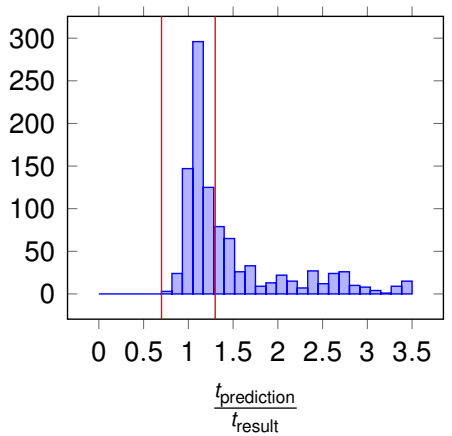

(f) Simple Model

- $0.7<\frac{t_{\text {tredidion }}}{t_{\text {tesult }}}<1.3$ for $71 \%$ of all samples for out model.

- $0.7<\frac{t_{\text {preadition }}}{t_{\text {tresut }}}<1.3$ for $61 \%$ of all samples for the simpler model. 


\section{Further Work}

- Improve predictions, expand onto more architectures

- Support more language constructs, e.g. if or for

- Support intrinsic operations, e.g. $\sin ()$, sqrt () 


\section{Thank you for your attention}

Acknowledgements This work was partly supported by the German Research Foundation (DFG) as part of the Transregional Collaborative Research Centre "Invasive Computing" (SFB/TR 89). 